\title{
Multiple Sites of Thrombosis Without Thrombocytopenia After a Second Dose of Pfizer-BioNTech COVID-19 Vaccine
}

Roberto Scendoni ( $\nabla$ roberto.scendoni@gmail.com )

ASUR Area Vasta 3 Macerata https://orcid.org/0000-0003-1910-2405

Cristina Petrelli

Ospedale di Macerata, Neurological Unit

Mauro Giustozzi

Ospedale di Macerata, Emergency Department

Francesco Ottavio Logullo

Ospedale di Macerata, Neurological Unit

\section{Case report}

Keywords: COVID-19 infection, Pfizer-BioNTech vaccine, Venous thrombosis, Thromboembolism, Thrombocytopenia, Computed tomography

Posted Date: September 28th, 2021

DOI: https://doi.org/10.21203/rs.3.rs-910520/v1

License: (c) (i) This work is licensed under a Creative Commons Attribution 4.0 International License. Read Full License 


\section{Abstract}

\section{Background}

In the current international scientific panorama rare cases of venous thrombotic complications following mRNA vaccine administration have been reported, consisting of cerebral sinus thromboses and acute venous thromboembolism.

\section{Case presentation}

The present paper describes the case of a 75-year-old woman in good health who developed cerebral venous thrombosis (CVT), deep venous thrombosis (DVT) and bilateral pulmonary emboli (PE) after receiving a second dose of Pfizer-BioNTech COVID-19 vaccine. A series of laboratory tests performed during the hospitalization yielded interesting results, allowing us to exclude thrombophilic risk factors and certify the absence of thrombocytopenia in the patient.

\section{Conclusion}

Although COVID-19 vaccination is the most important tool in stopping the pandemic, pharmacovigilance is crucial for detecting potential multisystem thrombotic events, even for mRNA vaccines.

\section{Highlights}

1. Thrombotic complications following mRNA COVID-19 vaccine administration are possible

2. Thrombosis without thrombocytopenia can occur after Pfizer-BioNTech vaccine

3. Multiple sites of thrombosis confirm that the vaccine-related mechanism is systemic

4. Further studies are needed to investigate the thrombotic etiopathogenesis

\section{Introduction}

COVID-19 vaccination campaigns with several vaccine types are currently underway. In extremely rare cases, the Johnson \& Johnson/Janssen and AstraZeneca COVID-19 vaccinations may cause vaccine-induced thrombotic thrombocytopenia (VITT) [1], a condition characterized by simultaneous acute thrombosis and thrombocytopenia. The condition is similar to heparin-induced thrombocytopenia. Given the extremely low case count, specific risk factors for VITT are yet to be determined, though onset seems to occur between 5 and 28 days post vaccination.

The rare risk of blood clots associated with the DNA vaccine is now well documented, but very recently concerns have begun to emerge about the mRNA COVID-19 vaccine [2].

A recent study [3] investigated the occurrence of venous thromboembolism (VTE) in three women following administration of the Moderna vaccination at the same healthcare system. None of them showed thrombocytopenia.

Here, we present a case of multiple sites of thrombosis without thrombocytopenia after a second dose of PfizerBioNTech COVID-19 vaccine. 


\section{Case Report}

We present the case of a 75-year-old woman in good health, with the exception of mild arterial hypertension, for which she was being treated with antihypertensive drugs. On entering the emergency medicine unit, the patient presented with acute onset of nonfluent aphasia and code stroke protocol was followed. The subject's past medical history was negative, except for the administration of a second dose of Pfizer-BioNTech COVID-19 vaccine (Fig. 1) 10 days before symptom onset.

In fact, two days after receiving the second dose the patient began to experience a persistent and drug-resistant headache, and focal neurological symptoms appeared approximately nine days after the aforementioned headache. In the emergency room a computed tomography (CT) scan of the head was performed with evidence of intraparenchymal hemorrhage in the left occipital lobe associated with perilesional vasogenic edema and further hemorrhagic lesions in the ipsilateral subcortical temporo-occipital site, with a round morphology.

The neurological physical examination showed nonfluent aphasia, but no sensory-motor deficits in the four limbs. CT images of the brain were sent to the on-call neurosurgeon who recommended computed tomography angiography (CTA) of intracranial circulation and antiedema therapy with mannitol and steroids.

After about 12 hours a brain CT scan was performed, which showed a significant deterioration, with numerical and dimensional increases in the hemorrhagic components of the temporal, occipital, and left parietal lobes with marked signs of perilesional edema. The CT image was also characterized by widespread hypovisualization of the cortical folds in the left hemispheric area due to the presence of edema, together with marked compression phenomena affecting the anterior and posterior horn and the trine of the left ventricular system. In the left frontoparietal region there was a subdural hematoma $(\mathrm{SDH})$ with a maximum thickness of $3 \mathrm{~mm}$.

The CTA showed evidence of extensive opacification defects affecting the left transverse sinus, the left sigmoid sinus, and the lower portion of the petrous rock of the left internal jugular vein, compatible with acute cerebral venous thrombosis (CVT). In addition, a focal filling defect of similar significance was also found in the right transverse sinus.

The patient was hospitalized in the neurology unit, where the following was prescribed: antiepileptic therapy with $500 \mathrm{mg}$ of levetiracetam twice a day; antiedema therapy with $50 \mathrm{cc}$ of mannitol four times a day for four days and subsequently $100 \mathrm{cc}$ three times a day until suspension. In addition, anticoagulant therapy with enoxaparin was administered at a dosage of $6000 \mathrm{IU}$ in the morning and $8000 \mathrm{IU}$ in the evening.

Magnetic resonance imaging (MRI) of the brain revealed an extensive left temporo-parieto-occipital edema, corresponding to an extensive area of intraparenchymal bleeding, and confirming evidence of thrombosis of the left transverse and sigmoid sinuses of the proximal tract of the left internal jugular.

In the days that followed, the patient underwent a comprehensive series of laboratory and instrumental examinations, aimed at finding the etiology of cerebral thrombosis. Routine laboratory tests consisted of a complete blood count, coagulation screening including factors II and V Leiden, checks for the presence of neoplastic markers, and measurements of homocysteine, ferritin, beta-2-microglobulin and immunoglobin levels. There was an increase of the D-dimer $(6175 \mathrm{ng} / \mathrm{ml}$ and $2885 \mathrm{ng} / \mathrm{ml}$ on the 4th and 9th day of hospitalization respectively), while the remaining parameters were within normal range, including the platelet count (Table 1). 
Table 1

Routine laboratory tests performed during hospitalization.

\begin{tabular}{|c|c|c|c|c|c|c|c|}
\hline & $18 / 05 / 2021$ & $20 / 05 / 21$ & $21 / 05 / 21$ & $22 / 05 / 21$ & $24 / 05 / 21$ & $27 / 05 / 21$ & $31 / 05 / 21$ \\
\hline $\begin{array}{l}\text { Leukocytes } \\
\text { (thousand/ } / \mathrm{mm}^{3} \text { ) }\end{array}$ & 7.6 & 15.2 & 13.1 & 8.7 & 7.9 & 6.7 & 4.9 \\
\hline $\begin{array}{l}\text { Erythrocytes } \\
\left(\text { million } / \mathrm{mm}^{3} \text { ) }\right.\end{array}$ & 4.23 & 4.20 & 4.22 & 4.2 & 4.34 & 4.11 & 3.95 \\
\hline $\begin{array}{l}\text { Hemoglobin } \\
(\mathrm{gr} / \mathrm{dl})\end{array}$ & 13.1 & 12.9 & 12.7 & 12.8 & 13.4 & 12.4 & 12.0 \\
\hline Hematocrit (\%) & 38.7 & 37.4 & 37.8 & 37.8 & 38.3 & 37.7 & 36.2 \\
\hline $\begin{array}{l}\text { Platelets } \\
\text { (thousand } / \mathrm{mm}^{3} \text { ) }\end{array}$ & 256 & 246 & 205 & 209 & 212 & 235 & 270 \\
\hline I.N.R. & 0.96 & / & / & l & 1.10 & 1.08 & 1.05 \\
\hline PTT RATIO & 0.80 & / & / & / & 0.86 & 0.81 & 0.91 \\
\hline $\begin{array}{l}\text { Fibrinogen } \\
(\mathrm{mg} / \mathrm{dl})\end{array}$ & 328 & / & I & / & 273 & 370 & 384 \\
\hline
\end{tabular}

A total body CT excluded neoplastic lesions, but a pulmonary thromboembolism with filling defects in both lower lobes was found. For this reason, a venous doppler examination of the lower limbs was carried out, with evidence of voluminous deep venous thrombosis (DVT) of a peroneal vessel of the left lower limb, extending to the distal lower third of the leg up to the tibial-peroneal trunk.

During hospitalization the patient presented progressive improvement of the language impairment with regard to anomic aphasia and semantic deficit. A subsequent brain CT revealed initial phenomena of hemoglobin degradation and reduction of blood hyperdensity on the left temporo-parietal intraparenchymal hemorrhagic focus. A marked edema and hypodensity area from hypovascularization of the thrombotic area persisted.

The patient was discharged after 15 days of recovery. Two weeks after discharge another brain CT showed a complete resorption of the hemorrhagic areas, and a CTA showed post embolic rehabilitation of the petrous tract of the internal jugular vein, and persisting stable filling defects from subtotal thrombosis affecting the transverse and sigmoid sinus. In addition, a Doppler examination of the lower limbs showed complete resolution of the venous thrombosis. The patient's therapy was changed from subcutaneous enoxaparin to warfarin, adjusted to obtain a correct PT/INR range.

\section{Discussion}

In this report we present a case of multiple thrombosis (cerebral, pulmonary, and lower limbs) occurring after a second dose of Pfizer-BioNTech COVID-19 vaccine. While risk of thrombosis following COVID-19 infection and potential thrombotic risks following both the AstraZeneca and Janssen COVID-19 vaccinations are now better documented, there have been very few cases of thrombosis after Pfizer-BioNTech and Moderna vaccines. The first case presented a popliteal and peroneal deep venous thrombosis after the second dose of the Pfizer-BioNTech 
vaccine, and the individual was found to be a carrier of the heterozygous factor $\mathrm{V}$ Leiden mutation; the second case related to three patients who developed acute venous thrombosis after the Moderna vaccine, without other apparent risk factors. Furthermore, this case is the second in the literature of cerebral venous sinus thrombosis temporally correlated with a vaccine, in particular Pfizer-BioNTech; the first case of this kind was documented after Moderna vaccine [4].

The peculiarity of our case is the absence of thrombocytopenia, which therefore rules out VITT. In addition, the absence of risk factors and potentially thrombotic drugs should be noted. In the case of documented thrombosis in a region after vaccine administration, it is necessary and mandatory to investigate whether other sites are affected by this pathology. The possible coexistence of thrombosis in different sites would confirm that the underlying mechanism is systemic. This highlights the importance of conducting an accurate and thorough examination when the first symptoms appear.

Although it is impossible to establish the exact process that led to multisite venous thrombosis, the timing of onset indicates a concrete correlation with the COVID-19 vaccine itself. Therefore, the hypothesis that the mRNA vaccine may activate a clotting mechanism should be taken into consideration. Given the normal platelet count of the hospitalized patient in our report, it would also be reasonable to investigate possible stress, triggered by the vaccine, on the vascular endothelium, which in normal conditions prevents hypercoagulability by releasing antithrombotic and anti-inflammatory factors, thus maintaining a certain level of blood fluidity [5].

\section{Conclusion}

Although COVID-19 vaccination is key to stopping the pandemic, ongoing pharmacovigilance is crucial to monitor potential side effects, thereby ensuring complete and accurate communication between healthcare vaccinator and patient. It is imperative to obtain a vaccination history in patients presenting symptoms of thrombosis with no other obvious predisposing factors.

\section{Declarations}

\section{Ethics approval and consent to participate}

The research fully adhered to ethical principles

\section{Consent for publication}

The patient's permission to present the case has been regularly obtained.

\section{Availability of data and material}

Data were obtained and are available from the archive of Macerata Hospital.

\section{Competing interests}

The authors declare that there is no conflict of interest regarding the publication of this report.

\section{Funding}


This research did not receive any specific grant from funding agencies in the public, commercial, or not-for-profit sectors.

\section{Author Contributions}

Conceptualization: R.S., methodology and formal analysis: C.P., investigation: M.G., resources and supervision: F.O.L. All authors have read and agreed to the published version of the manuscript.

\section{Acknowledgements}

Thanks to Jemma Dunnill for proofreading the manuscript.

\section{References}

1. Greinacher A, Thiele T, Warkentin TE, Weisser K, Kyrle PA, Eichinger S (2021) Thrombotic thrombocytopenia after ChAdOx1 nCov-19 vaccination. N Engl J Med 384:2092-2101.

2. Carli G, Nichele I, Ruggeri M, Barra S, Tosetto A (2021) Deep vein thrombosis (DVT) occurring shortly after the second dose of mRNA SARS-CoV-2 vaccine. Intern Emerg Med 16(3):803-804.

3. Andraska EA, Kulkarni R, Chaudhary M, Sachdev U (2021) Three cases of acute venous thromboembolism in females following vaccination for COVID-19. J Vasc Surg Venous Lymphat Disord S2213-333X(21):00392-9.

4. Sangli S, Virani A, Cheronis N, Vannatter B, Minich C, Noronha S, Bhagavatula R, Speredelozzi D, Sareen M, Kaplan RB (2021) Thrombosis with thrombocytopenia after the messenger RNA-1273 vaccine. Ann Intern Med L21-0244.

5. van Hinsbergh VW (2012) Endothelium--role in regulation of coagulation and inflammation. Semin Immunopathol 34(1):93-106.

\section{Figures}


PREN. EFORMQ5PP89WW DEL 07/05/2021
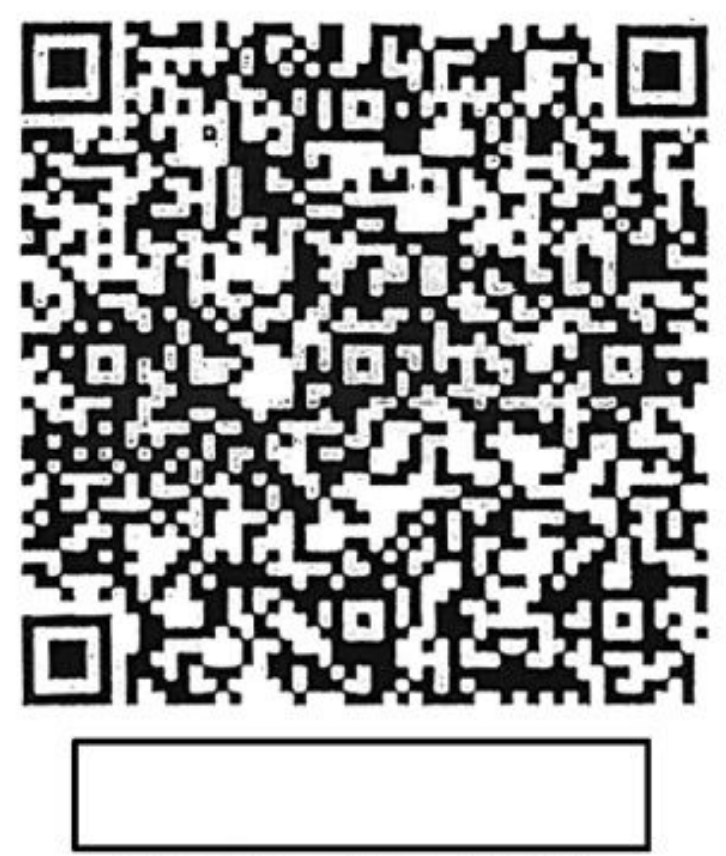

TIPO VACCINO: PFIZER

CODICE: FA5831

Figure 1

Patient's Pfizer-BioNTech COVID-19 vaccination QR code relating to the second dose administered May 7, 2021. 